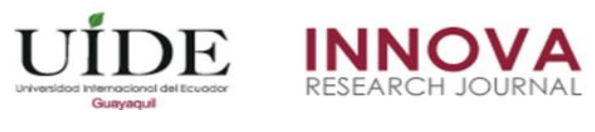

INNOVA Research Journal, ISSN 2477-9024

(Mayo, 2018). Vol. 3, No.5 pp. 89-98

DOI: https://doi.org/10.33890/innova.v3.n5.2018.679

URL: http://revistas.uide.edu.ec/index.php/innova/index

Correo: innova@uide.edu.ec

\title{
Análisis ergonómico con el método checklistocra en trabajadores de una industria alimentaria
}

\section{Ergonomic analysis with the checklistocra method in workers of a food industry}

Cristian Laverde Albarracín.

Universidad Tecnológica Equinoccial, Ecuador

Universidad Técnica Estatal de Quevedo, Ecuador

Marco Vinicio Noroña Merchán

Universidad Internacional del Ecuador, Ecuador

Ruth Torres

Irene Bustillos Molina

Universidad Técnica Estatal de Quevedo, Ecuador

Autor para correspondencia: cristian.laverde@ute.edu.ec; claverde@uteq.edu.ec

Fecha de recepción: 25 de Octubre de 2017 - Fecha de aceptación: 15 de Mayo de 2018

Resumen: Los trastornos producidos por movimientos repetitivos, producen serias afectaciones a la salud de los trabajadores, la investigación se realizó en el personal de la línea de faenamiento de reses de una empresa de Santo Domingo de los Tsáchilas de junio a septiembre del 2017 a fin de evaluar los movimientos repetitivos (MR) de miembros superiores, sus características, relación con condiciones laborales y otros factores. Se utilizó como herramienta, el análisis ergonómico del puesto, la aplicación del método ergonómico CheckListOcra y el examen médico específico. En el puesto de sangría se realizó la evaluación, donde se cumplen actividades semejantes al resto de puestos de la línea. Se identificaron 11 ciclos de acciones, todos realizan movimientos repetitivos al igual que otros riesgos ergonómicos, también se jerarquizaron las etapas de trabajo donde las actividades repetitivas fue mayor en frecuencia en las acciones 6, 8,9 y 11 con duración de 3 segundos. Se corroboró con el análisis médico específico a los 55 empleados y se reportó que el $40 \%$ están positivos a la exploración física, el 56\% a la exploración neurológica y el método ergonómico determinó riesgos altos para la extremidad superior derecha y medio en la extremidad superior izquierda

Palabras Claves: condiciones de trabajo; línea de faenamiento; movimiento repetitivo; trastornos musculoesqueléticos

\begin{abstract}
The disorders produced by repetitive movements, produce serious damages to the health of the workers, the investigation was carried out in the personnel of the line of slaughter of cattle of a company of Santo Domingo de los Tsáchilas from June to September of 2017 in order to evaluate repetitive movements (MR) of upper limbs, their characteristics, relationship with working conditions and other factors. It was used as a tool, the ergonomic analysis of the position, the application of the ergonomic method CheckListOcra and the specific medical examination. In the indentation position, the evaluation was carried out, where activities similar to the rest of the
\end{abstract}


posts in the line are carried out. We identified 11 cycles of actions, all performed repetitive movements as well as other ergonomic risks, we also ranked the work stages where the repetitive activities were higher in frequency in actions $6,8,9$ and 11 with a duration of 3 seconds. Corroborated with the specific medical analysis to the 55 employees and it was reported that $40 \%$ are positive to the physical examination, $56 \%$ to the neurological examination and the ergonomic method determined high risks for the right upper extremity and middle in the upper extremity left. Key Words: working conditions; slaughter line; repetitive motion; musculoskeletal disorders

\section{Introducción}

Uno de los mayores retos de la ergonomía ha sido el estudio de la interacción del hombre frente a los requerimientos físicos (postura, fuerza, movimiento). Cuando estos requerimientos sobrepasan la capacidad de respuesta del individuo o no hay una adecuada recuperación biológica de los tejidos, este esfuerzo puede asociarse con la presencia de lesiones músculoesqueléticas relacionadas con el trabajo (Vernaza-Pinzon 2005). Es evidente, que la mayoría de las lesiones músculo-esqueléticas no se producen por accidentes o por agresiones únicas o aisladas, sino como resultado de pequeños y repetidos traumatismos, por ello se han venido denominando como traumatismos repetitivos o acumulativos, o con otros acrónimos anglosajones como WMSD (Work-MusculoskeletalDisorders), CTD (Cumulative Trauma Disorders),RSI (RepetitiveStrainInjury), incluso se han aceptado síndromes de nuevo cuño como es el caso de cervicobraquialgia ocupacional u OCD (OcupationalOveruseSyndrome) (Vispe, Roman 2007).

Estos trastornos, de aparición lenta y de carácter inofensivo en apariencia, se localizan fundamentalmente en los tendones y sus vainas, y pueden también dañar o irritar los nervios, o impedir el flujo sanguíneo por compresión de los pequeños vasos que nutren los tejidos. Son frecuentes en la zona de la mano-muñeca-brazo, y en el hombro y cuello. En otras ocasiones serán las estructuras óseas, fundamentalmente a nivel de la columna lumbar las que resultarán dañadas (Bascuas 2012).

La Empresa donde se realizó la investigación, es una planta de faenamiento de reses, ubicada en Santo Domingo de los Tsàchilas, donde prestan sus servicios 103 empleados distribuidos en diferentes áreas de trabajo. En la línea de faenamiento el número de trabajadores es de 55, repartidos en 12 etapas del proceso que son; aturdimiento, izado, sangrado, descuerado, eviscerado, cortes, limpieza de canal, pesaje-calificación-almacenamiento, proceso de vísceras blancas, proceso de vísceras rojas, proceso de cabeza y proceso de patas. Los empleados realizan jornadas de trabajos de 8 horas durante la mañana y tarde, además horas extras de ser necesario, de lunes a viernes cumpliendo 40 horas semanales.

En todas las etapas de la línea de faenamiento se cumplen actividades donde realizan movimientos repetitivos de miembros superiores (flexión, extensión, abducción, aducción, rotación interna, rotación externa) involucrando a hombro, codo, muñeca, mano y dedos. Movimientos de columna (flexión, extensión, desviación lateral, rotación), además permanecen de pie por tiempos prolongados, en posiciones incómodas durante toda la jornada laboral.

El proceso de faenamiento está sincronizado por la máquina en un tiempo de 55 segundos por cada res, dificultando el cumplimiento de las pausas laborales. Este estudio espera aportar 
evidencias de la existencia de TME de extremidades superiores en el personal de una empresa de faenamiento de reses en Santo Domingo de los Tsàchilas Ecuador.

\section{Objetivo}

Debido a que los procesos dentro del faenamiento de reses, por la naturaleza propia del trabajo tienen movimientos repetitivos en extremidades superiores, es de interés el estudio ya que permite conocer como es la afectación en la salud de los trabajadores expuestos a este factor de riesgo, las conclusiones de la investigación, permitirán tomar medidas para mejorar las condiciones laborales de los trabajadores.

\section{Material y métodos}

Se realizó una investigación descriptiva de diseño transversal en la población de la línea de faenamiento durante junio a septiembre del 2017, con una participación de 55 empleados (100\%). Se utilizó como herramienta, el análisis ergonómico del puesto, la aplicación del método ergonómico CheckListOcra y el examen médico específico. Se realizó una exploración Neurológica por medio de los siguientes Test y Signos

- Test de Nerr,

- Test de Tinel,

- Test de Phalen

- Signo de Yergason

Con la exploración se determinó el nivel de afectación a la salud de los trabajadores expuestos al factor de riesgo evaluado con la metodología descrita. Adicionalmente, se visitaron todas las áreas de la línea de faenamiento de la Empresa, en horas laborables, con la finalidad de observar el trabajo que realizan los empleados.

\section{Resultados}

La población estudiada fue de 55 empleados, todos de sexo masculino. En la tabla 1 se pueden ver las principales características del personal que labora en la línea de faenamiento. El $100 \%$ del personal está distribuido en las 15 secciones como se indica en la tabla 2 que forman todo el proceso de faenamiento de res (aturdimiento, izado, sangría, descuerado, eviscerado, cortes y limpieza de canal, pesaje, calificación, almacenamiento, zona de vísceras blancas, vísceras rojas, descuerado de cabeza, proceso de patas, deshuese de cabeza, transporte de cabeza).

Tabla 1. Características de los empleados de la línea de faenamiento de una empresa en Santo Domingo. Ecuador. Junio-Septiembre, 2017

\begin{tabular}{lc}
\hline \multicolumn{1}{c}{ Características } & Resultados \\
\hline Tamaño de la Muestra & 55 \\
Edad (Promedio, años) & 31,8 \\
Peso (Promedio, Kg) & 74,7 \\
Altura(Promedio, cm) & 164,0 \\
IMC (Promedio, Kgm2) & 27,7 \\
Lateralidad (\%, diestras) & 100 \\
\hline
\end{tabular}




$\begin{array}{ll}\text { Traumas anteriores }(\%) & 16 \\ \text { No Traumas anteriores }(\%) & 84 \\ \text { Actividad extra laboral (\%) } & 71 \\ \text { No Actividad extra laboral (\%) } & 29\end{array}$

Tabla 2 Distribución de los empleados de la línea de faenamiento de una empresa en Santo Domingo. Ecuador. Junio-Septiembre, 2017

\begin{tabular}{lc}
\hline \multicolumn{1}{c}{ ÁREAS } & $\mathrm{N}^{\circ}$ EMPLEADOS \\
\hline Aturdimiento & 1 \\
Izado & 2 \\
Sangría & 1 \\
Descuerado & 9 \\
Eviscerado & 5 \\
Cortes y limpieza de canal & 4 \\
Pesaje & 2 \\
Calificación & 2 \\
Almacenamiento & 2 \\
Vísceras blancas & 9 \\
Vísceras rojas & 3 \\
Descuerado de cabeza & 6 \\
Proceso de patas & 2 \\
Deshuese de cabeza & 4 \\
Transporte & 3 \\
\hline
\end{tabular}

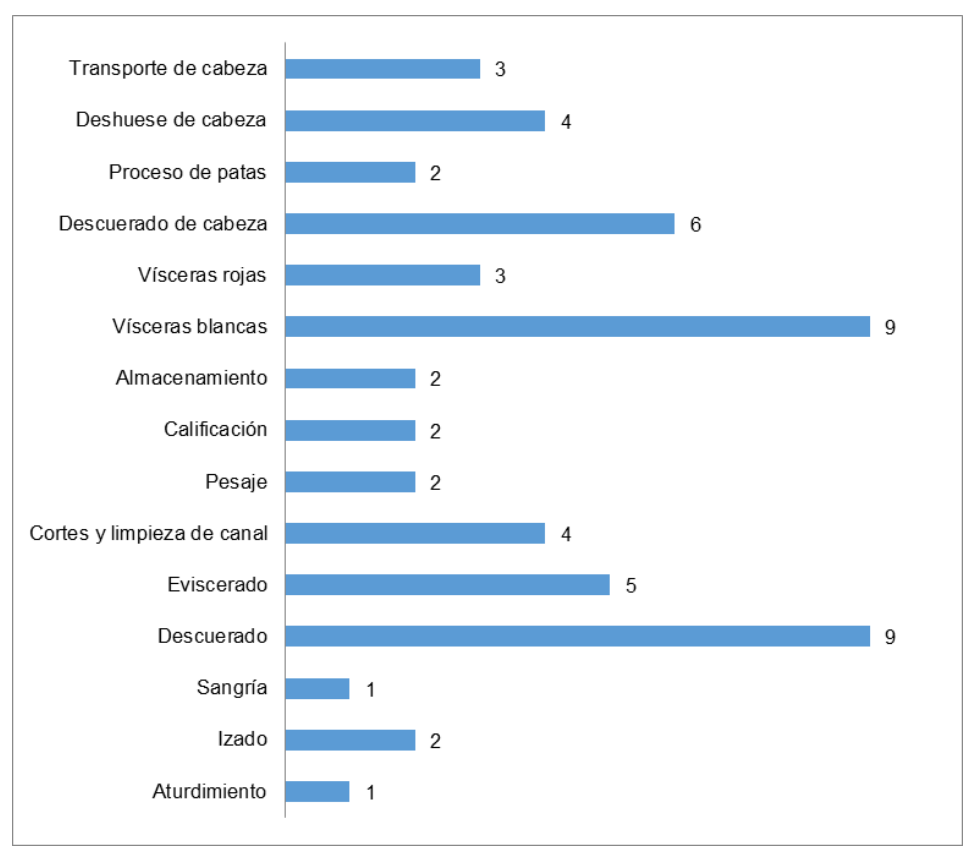

Figura 1 Distribución de los empleados de la línea de faenamiento de una empresa en Sto. Dgo. Ecuador. JunioSeptiembre, 2017

Tabla 3. Exploración Física Personal de la línea de Faenamiento Santo Domingo- Ecuador. Junio-septiembre 2017

\begin{tabular}{lll}
\hline Positivos & 22 & $40 \%$ \\
Negativos & 33 & $60 \%$ \\
\hline
\end{tabular}




\begin{tabular}{lcc}
\hline & Movimiento-Dolor Hombro & \\
Derecho & 10 & $18 \%$ \\
Izquierdo & 4 & $7 \%$ \\
Bilateral & 4 & $7 \%$ \\
Total & 18 & $32 \%$ \\
& Movimiento-Dolor Codos & \\
Derecho & 4 & $7 \%$ \\
Izquierdo & 2 & $4 \%$ \\
Bilateral & 2 & $4 \%$ \\
Total & 8 & $15 \%$ \\
& 4 & \\
Derecho & Movimiento-Dolor Muñeca & $7 \%$ \\
Izquierdo & 2 & $4 \%$ \\
Bilateral & 1 & $2 \%$ \\
Total & 7 & $13 \%$ \\
& 4 & $7 \%$ \\
Derecho & Movimiento-Dolor Manos & $2 \%$ \\
Izquierdo & 1 & $2 \%$ \\
Bilateral & 1 & $11 \%$ \\
Total & 6 & \\
\hline
\end{tabular}

\section{Exploración neurológica: signos y test}

Tabla 4. Exploración Neurológica Personal de la línea de Faenamiento Santo DomingoEcuador. Junio-septiembre 2017.

\begin{tabular}{|c|c|c|}
\hline Positivos & 31 & $56 \%$ \\
\hline Negativos & 24 & $46 \%$ \\
\hline \multicolumn{3}{|c|}{ Test de Neer } \\
\hline \multicolumn{3}{|l|}{ Derecho } \\
\hline Izquierdo & \multicolumn{2}{|r|}{$1.82 \%$} \\
\hline Bilateral & \multicolumn{2}{|r|}{$7.27 \%$} \\
\hline \multicolumn{3}{|c|}{ Signo de Yergason } \\
\hline Derecho & & $3.63 \%$ \\
\hline Izquierdo & & $1.81 \%$ \\
\hline Bilateral & & $3.63 \%$ \\
\hline \multicolumn{3}{|c|}{ Epicondilitis } \\
\hline \multicolumn{3}{|r|}{$14.55 \%$} \\
\hline Izquierdo & \multicolumn{2}{|r|}{$7.27 \%$} \\
\hline Bilateral & & $12.73 \%$ \\
\hline \multicolumn{3}{|c|}{ Epitrocleitis } \\
\hline Derecho & \multicolumn{2}{|r|}{$3,63 \%$} \\
\hline Izquierdo & \multicolumn{2}{|r|}{$3.63 \%$} \\
\hline Bilateral & \multicolumn{2}{|r|}{$9.09 \%$} \\
\hline \multicolumn{3}{|c|}{ Filkekstein } \\
\hline Derecho & \multicolumn{2}{|r|}{$12.72 \%$} \\
\hline Izquierdo & \multicolumn{2}{|r|}{$9.09 \%$} \\
\hline Bilateral & & $7.27 \%$ \\
\hline \multicolumn{3}{|c|}{ Test de Phalen } \\
\hline Derecho & & $4,45 \%$ \\
\hline Izquierdo & & $3.63 \%$ \\
\hline Bilateral & & $3.63 \%$ \\
\hline \multicolumn{3}{|c|}{ Test de Tinel } \\
\hline Derecho & & $5,45 \%$ \\
\hline Izquierdo & & $1.82 \%$ \\
\hline Bilateral & & $1.82 \%$ \\
\hline
\end{tabular}




\section{Evaluación Ergonómica}

Tabla 5. Resultados de la evaluación del puesto de trabajo con Check List OCRA

\begin{tabular}{|c|c|c|c|c|}
\hline Extremidad & Evaluación & $\begin{array}{l}\text { Check List } \\
\text { Ocra }\end{array}$ & Riesgo & Acciones \\
\hline \multirow[t]{2}{*}{ Derecha } & Trabajador & 32.5 & Alto & $\begin{array}{l}\text { Se recomienda mejora del puesto, supervisión } \\
\text { médica y entrenamiento }\end{array}$ \\
\hline & Puesto & 35 & Alto & $\begin{array}{l}\text { Se recomienda mejora del puesto, supervisión } \\
\text { médica y entrenamiento }\end{array}$ \\
\hline \multirow[t]{2}{*}{ Izquierda } & Trabajador & 17.1 & Medio & $\begin{array}{l}\text { Se recomienda mejora del puesto, supervisión } \\
\text { médica y entrenamiento }\end{array}$ \\
\hline & Puesto & 18.5 & Medio & $\begin{array}{l}\text { Se recomienda mejora del puesto, supervisión } \\
\text { médica y entrenamiento }\end{array}$ \\
\hline
\end{tabular}

\section{Discusión}

Basados en la clasificación de la OMS en lo referente al IMC, de todos los empleados de la línea de faenamiento, el 38\% (21) presentaron un IMC dentro del rango normal. Sin embargo el 42\% (23) presentaron Pre-Obesidad, el 16\% (9) Obesidad Grado I, el 4\% (2) Obesidad Grado II (Gomez, Raygoza y Sandoval, 2017) . Es importante tomar en cuenta esta variable de peso, la altura y el IMC, ya que han sido identificadas como factores de riesgo potenciales de los TME, especialmente para el síndrome del túnel carpiano, motivo de otro estudio.

Además hay que tener presentes las variables de edad y talla, importantes como factores de riesgo de los TME, porque constituyen el problema de salud más importante entre los trabajadores de mediana y avanzada edad (Asensio 2012). Dentro de las actividades que cumplen en la línea de faenamiento el personal. El 56\% (31) están en edades entre 18 y 29 años, el 24\% (13) entre 30 y 39 años, el $9 \%$ (5) entre 40 y 49 años, $9 \%$ (5) entre 50 y 59 años y el 2\% (1) más de 60 años. En entornos laborales caracterizados por la existencia de trabajos repetitivos con ciclos de trabajos cortos, la edad parece influir en la asignación de los puestos a los trabajadores (Araña y Patten,2011). Existe una clara tendencia a que los puestos más favorables sean asignados a trabajadores de más edad (de más de 45 años), mientras que a los trabajadores más jóvenes se les asignan puestos más desfavorables (Arenas y Cantú 2011).

El 25\% (14) empleados están con una talla menor a 1,60 cm., el 55\% (30) entre 1,60 y 1,70 com., y el $20 \%$ (11) más de $1,70 \mathrm{~cm}$. Es necesario tener presente esta variable, hay puestos en las que el empleado no deber sobrepasar $1.70 \mathrm{~cm}$ de estatura, caso contrario tendría que realizar flexión de columna cervico-dorso-lumbar para cumplir la actividad y producir lesiones a ese nivel, o casos menor a 1,60 cm tendría que realizar hiperflexión, hiperextensión o hiperrotación de columna y extremidad superior, adoptando postura ergonómica incorrecta dando lugar a la presencia de lesiones músculo-esqueléticas de extremidad superior.

\section{Condiciones de trabajo}

La situación laboral, considerando que las actividades y tareas realizadas en el trabajo, así como su duración y frecuencia durante cada jornada laboral, pueden ayudar a explicar su 
asociación con los diversos TME (Vicente, 2008) Ante esto tenemos que el 100\% (55) cumplen jornada laboral de 8 horas diarias, pausa de 1 hora durante el almuerzo, turno de mañana - tarde y 40 horas semanales. El 42\% (23) cumplen rotación de menor a 3 meses, el 13\% (7) de 4 a 6 meses, el 9\% (5) cada 9 meses y el 36\% (20) cada año, variable desfavorable ya que no se está cumpliendo con la correcta rotación del puesto laboral. Se considera tiempo de recuperación el periodo de descanso que sigue a un periodo de actividad en el cual el grupo de músculos que normalmente están implicados en la tarea de trabajo están básicamente inactivos y por tanto puede darse el restablecimiento del tejido humano. La insuficiencia de tiempo para la recuperación del cuerpo entre movimientos repetidos aumenta el riesgo de trastornos músculoesqueléticos, por tanto el factor de riesgo es realmente la falta de tiempo de recuperación (Vispe, Roman 2007).

Desde el punto de vista fisiológico son mejores las pausas cortas pero frecuentes que las más largas y espaciadas. Según la Comisión para la Seguridad y Salud australiana (Muñoz y Velasco 2017), no son admisibles períodos de trabajo con movimientos repetidos que superen los 60 minutos sin períodos de recuperación. La proporción adecuada es 5: 1, es decir 10 minutos de recuperación por cada 50 trabajados (Gutierrez, Loéz y Ortega, 2017).

\section{Actividad Laboral}

Otro de los factores predisponentes como es el factor físico y biomecánico en la presencia de lesiones musculo-esqueléticas (Hernandez y Mateus, 2013), es conocer qué tipo de actividades cumplen los empleados en la línea de faena. El 100\% (55) realizan movimiento repetitivo de miembros superiores y el 56\% (31) realizan manipulación manual de cargas con peso mayor a $3 \mathrm{~kg}$, este último será de aplicación a cualquier trabajador, que tras la evaluación de riesgos en su puesto de trabajo se compruebe que manipula manualmente cargas, siempre que éstas superen los $3 \mathrm{~kg}$. de peso (Harari, 2010); entre 1 y $3 \mathrm{~kg}$ el 22\% (12), menos de 1 kilo el $22 \%$ (12). Factores desencadenantes de lesiones a nivel de miembros superiores.

\section{Antecedentes Laborales}

Conocer actividades anteriores relacionadas a movimientos repetitivos y extra laborales (tabla 1) ayuda a identificar las posibles causas de las lesiones musculo-esqueléticas de miembros superiores; inciden en alto porcentaje en el diagnóstico definitivo de la lesión (Medina y Castillo 2013). El 64\% (35) si han estado expuestos a movimiento repetitivo de miembros superiores y el 36\% (20) no ha estado expuesto. El 29\% (16) si realizan actividades extra laborales, en mayor porcentaje labores agrícolas y el 71\% (39) no realizan actividades fuera del horario de trabajo de la empresa.

\section{Antecedentes Médicos o Patológicos}

Es importante saber si los empleados de la línea de faenamiento a través de la historia clínica tienen antecedentes anteriores de lesiones osteomusculares en miembros superiores, antecedentes traumáticos, cirugías, alergias, enfermedades congénitas, enfermedades y tratamientos actuales (Vicente, 2008). El 44\% (24) tienen antecedente de lesión en miembros superiores y el 56\% (31) no tiene ningún antecedente; el 16\% (9) si han presentado 
anteriormente algún tipo de trauma en miembros superiores y el 84\% (46) no han sufrido ningún trauma anterior (tabla1); el 2\% (1) si tiene antecedente de enfermedad crónica y el 98\% (54) no tiene ningún tipo de antecedente.

\section{Exploración Física: Movimiento - dolor en las extremidades superiores}

El 40\% (22) están positivos y el 60\% (33) negativos (tabla 3). Para valorar la presencia o no de dolor de extremidad superior se realizaron movimientos del hombro flexión-extensión, abducción-aducción, rotación interna-rotación externa. El resultado del análisis determinó que del total de positivos (18) el 18\% (10) están positivos de dolor a nivel de hombro derecho, el7\% (4) hombro izquierdo y el 7\% (4) bilateral. A los movimientos de codo de flexión-extensión, supinación-pronación se determinó que del total de positivos $15 \%$ (8) el 7\% (4) positivos de dolor a nivel de codo derecho, el 4\% (2) a nivel de codo izquierdo y el 4\% (2) a nivel bilateral. A los movimientos muñeca de flexión-extensión, desviación radial y desviación cubital están positivos un total del $13 \%$ (7) de los cuales el $7 \%$ (4) corresponden al lado derecho, 4\% (2) lado izquierdo y el 2\% (1) ambos lados. A los movimientos de manos de flexión-extensión, desviación radial y desviación cubital están positivos un total del 11\% (6), con un 7\% (4) al lado derecho, un $2 \%$ (1) lado izquierdo y un $2 \%$ (1) bilateral.

\section{Exploración Neurológica, Test y Signos}

La exploración neurológica determinó que el 56\% de los empleados son positivos al análisis con los signos y test (Tabla 4). El más alto número de casos positivos fue para la valoración de epicondilitis con 14,55 \% de casos positivos en el lado derecho, 7,27 \% en el lado izquierdo y $12,73 \%$ en ambas extremidades; el signo de filkelstein determinó $12,73 \%$ de casos positivos para el lado derecho, 9,09 \% en el lado izquierdo y 7,27 \% bilateral; el test de Nerr presentó casos positivos en 9,09\% de casos en lado derecho, 1,82 \% en lado izquierdo y 7,27 \% casos bilateral; el test de Tinel presentó valores positivos más bajos, siendo el 5,45\% de casos positivos en lado derecho, $1,82 \%$ en lado izquierdo y $1,82 \%$ bilateral; los casos de epitrocleìtis están positivos en un 3.63\% para el lado derecho e izquierdo y un 9,09 bilateral; con menor afectación está el test de Phalen 4,45\% lado derecho, 3,63\% lado izquierdo y bilateral; por último el signo de Yergason determinó el 3,63 lado derecho, 1,81 lado izquierdo y 3,63 ambas extremidades. En el análisis se observa que la extremidad más afectada es el lado derecho, esto se debe a mayor movimiento repetitivo de esta extremidad a los que están expuestos los empleados y la ausencia de pausas de los trabajadores (micro pausas) que puedan evitar las lesiones.

\section{Evaluación de Riesgo Ergonómico}

Los riesgos en el puesto de trabajo de faenamiento de reses mediante la evaluación con el método CheckListOcra son altos para la extremidad superior derecha y medio en la extremidad superior izquierda, debido a la rapidez que se debe realizar las labores en cada una de las etapas de trabajo. Este método tiene como objetivo alertar sobre posibles trastornos, principalmente de tipo músculo-esqueléticos (TME) derivados de una actividad repetitiva (Marquez y Marquez, 2015). 
Estos resultados permiten adoptar medidas organizativas tales como rotaciones o pausas más frecuentes en el puesto de trabajo, de forma provisional o definitivamente, para evitar lesiones. Formación de los trabajadores e información para los riesgos laborales y las medidas de prevención. Control de la eficacia de la información y formación a los trabajadores (Uribe y Bernal, 2018).

\section{Conclusión}

De acuerdo al método CheckListOcra y la valoración neurológica, la carga causada por los Movimientos Repetitivos de miembros superiores, en los trabajadores del área de faenamiento de reses, tiene efectos nocivos, sobre el sistema musculo esquelético, comprendiendo un riesgo laboral que perjudica directamente a la salud y limitando su actividad laboral.

\section{Bibliografía}

Araña-Suárez, M., \& Patten, S. B. (2011). Trastornos músculo-esqueléticos, Psicopatología y Dolor. Trastornos Musculoesqueléticos Psicopatología, 1.

Asensio-Cuesta, S., Ceca, M. J. B., \& Más, J. A. D. (2012). Evaluación ergonómica de puestos de trabajo. Editorial Paraninfo.

Arenas-Ortiz, L., \& Cantú-Gómez, Ó. (2013). Factores de riesgo de trastornos músculoesqueléticos crónicos laborales. Medicina Interna de México, 29(4), 370-379.

Bascuas Javier; Hueso, Rosa. (2001). Ergonomía: 20 preguntas básicas para aplicar la ergonomía en la empresa, (2 ed.). Madrid: Fundación Mapfre.

Harari, F. (2010). Trastornos músculo-esqueléticos en auxiliares de enfermería de un hospital en Quito. Eídos, (3), 30-43.

Hernández, J. R. C., \& Mateus, H. O. (2013). Incorporación de Riesgos Ergonómicos en el Balanceo de Líneas de Ensamble en U. WPOM, 4(2), 29-43.

Gómez, B. L. L., Raygoza, N. P., \& Sandoval, S. D. C. D. (2017). Relación de la percepción del estilo de vida con sobrepeso/obesidad en adultos jóvenes con antecedentes familiares de diabetes mellitus tipo 2. Jóvenes en la ciencia, 3(2), 193-197.

Gutiérrez Muñoz, P., López Henao, Y., Ortega, W. S., Valencia, A. P., \& Santa, J. S. (2017). Comportamiento de la carga física en miembros superiores del personal operativo del área de confección de industrias Mavitex SAS.

Márquez Gómez, M., \& Márquez Robledo, M. (2015). Factores de riesgo biomecánicos y psicosociales presentes en la industria venezolana de la carne. Ciencia \& trabajo, 17(54), 171-176. 
Medina, M., \& Castillo, J. A. (2013). Evaluación de los desórdenes musculoesqueléticos en una línea de producción de alimentos. Análisis comparado de la postura y de la actividad de trabajo usando 4 métodos. Fisioterapia, 35(6), 263-271.

Muñoz, M. A., \& Velasco, Y. 2015. Evaluación de posturas de trabajo en la actividad de archivar documentos de proyectos de investigación. Universidad, Ciencia y Tecnología, 19(76), 128-137.

Uribe López, J. F., Bernal Osorio, M., \& Fernández Carrera, C. J. (2018). Aplicación del método" MAPO" para evaluar el riesgo biomecánico por movilización de pacientes en auxiliares de enfermería del Hospital General San Isidro de la ciudad de Manizales.

Vernaza-Pinzón, P., \& Sierra-Torres, C. H. (2005). Musculoskeletal pain and its association with ergonomic risk factors in administrative workers. Revista de Salud Pública, 7(3), 317-326.

Vicente, M., Ramírez, M., \& Murcia, J. (2008). Medicina del Trabajo, Protocolos y Prácticas de Actuación.

Vispe Román, C., Bascuas Hernández, J., Martínez Jarreta, B., \& Alcalde Lapiedra, V. (2007). Propuesta de un método de valoración de daño por lesión musculoesquelética como instrumento de prevención terciaria en el medio laboral. Mapfre Medicina, 18(1), 42-52. 